ANNALES

POLONICI MATHEMATICI

LXV.2 (1997)

\title{
On weak solutions of functional-differential abstract nonlocal Cauchy problems
}

\author{
by Ludwik BYszewski (Kraków)
}

\begin{abstract}
The existence, uniqueness and asymptotic stability of weak solutions of functional-differential abstract nonlocal Cauchy problems in a Banach space are studied. Methods of $m$-accretive operators and the Banach contraction theorem are applied.
\end{abstract}

1. Introduction. In this paper we study the existence, uniqueness and asymptotic stability of weak solutions of nonlocal Cauchy problems for a non-linear functional-differential evolution equation. Methods of $m$-accretive operators and the Banach contraction theorem are applied. The functionaldifferential problem considered here is of the form

$$
\begin{gathered}
u^{\prime}(t)+A(t) u(t)=f\left(t, u_{t}\right), \quad t \in[0, T], \\
u_{0}=g\left(u_{T^{*}}\right) \in C_{0} \subset C, \quad T^{*} \in\left[t_{0}+r, T\right],
\end{gathered}
$$

where for every $t \in[0, T], A(t): X \supset D(A(t)) \rightarrow X$ is an $m$-accretive operator, $X$ is a Banach space, $f:[0, T] \times C \rightarrow X, g: C \rightarrow C_{0}, u:$ $[-r, T] \rightarrow X, u_{t} \in C, t \in[0, T], C:=C([-r, 0], X), T>r>0$ and $t_{0}$ is a positive constant. Also, problems of type (1.1)-(1.2) on the interval $[0, \infty)$ are investigated.

The results obtained are generalizations of those given by Kartsatos and Parrott [8] on the existence and uniqueness of a weak solution of the Cauchy problem

$$
\begin{aligned}
u^{\prime}(t)+A(t) u(t) & =f\left(t, u_{t}\right), \quad t \in[0, T], \\
u_{0} & =\phi \in C_{0},
\end{aligned}
$$

and on the existence, uniqueness and stability of a weak solution of a problem of type (1.3)-(1.4) on the interval $[0, \infty)$.

1991 Mathematics Subject Classification: 47H06, 47H20, 34G20, 34K30, 34K25.

Key words and phrases: abstract Cauchy problems, functional-differential equation, nonlocal conditions, weak solutions, existence, uniqueness, asymptotic stability, $m$-accretive operators, Banach contraction theorem. 
The paper is a continuation of papers [2-4] on the existence and uniqueness of solutions of nonlocal Cauchy problems for evolution equations.

Theorems about the existence, uniqueness and stability of solutions of the abstract evolution Cauchy problem (1.3)-(1.4) in the differential version were studied by Bochenek [1], Crandall and Pazy [5], Evans [6] and Winiarska [9], [10].

2. Preliminaries. Let $X$ be a Banach space with norm $\|\cdot\|$ and let $C:=C([-r, 0], X)$, where $r$ is a positive number. The Banach space $C$ is equipped with the norm $\|\cdot\|_{C}$ given by the formula

$$
\|\psi\|_{C}:=\sup _{t \in[-r, 0]}\|\psi(t)\| \quad \text { for } \psi \in C .
$$

Let $T>r$ and let $t_{0} \in(a, T-r)$, where $a \geq 0$ will be defined in Section 4 .

For a continuous function $w:[-r, T] \rightarrow X$, we denote by $w_{t}$ the function belonging to $C$ and given by the formula

$$
w_{t}(\tau):=w(t+\tau) \quad \text { for } t \in[0, T], \tau \in[-r, 0] .
$$

An operator $B: X \supset D(B) \rightarrow X$ is said to be accretive (see [5]) if

$$
\left\|x_{1}-x_{2}+\lambda\left(B x_{1}-B x_{2}\right)\right\| \geq\left\|x_{1}-x_{2}\right\|
$$

for every $x_{1}, x_{2} \in D(B)$ and $\lambda>0$.

An accretive operator $B: X \supset D(B) \rightarrow X$ is said to be $m$-accretive (see $[6])$ if

$$
R(I+\lambda B)=X \quad \text { for all } \lambda>0,
$$

where $R(I+\lambda B)$ is the range of $I+\lambda B$.

We will need the following assumption:

Assumption $\left(\mathrm{A}_{1}\right)$. For each $t \in[0, T], A(t): X \supset D(A(t)) \rightarrow X$ is $m$-accretive, and there exist $\lambda_{0}>0$, a continuous nondecreasing function $l:[0, \infty) \rightarrow[0, \infty)$ and a continuous function $h:[0, T] \rightarrow X$ such that

$$
\begin{aligned}
& \left\|(I+\lambda A(t))^{-1} x-(I+\lambda A(s))^{-1} x\right\| \leq \lambda\|h(t)-h(s)\| l(\|x\|) \\
& \text { for all } \lambda \in\left(0, \lambda_{0}\right), t, s \in[0, T], x \in \overline{D(A(t))} .
\end{aligned}
$$

Assumption $\left(\mathrm{A}_{1}\right)$ implies that the set $\overline{D(A(t))}$ is independent of $t$ (see Lemma 3.1 of $[6])$. Therefore, we will denote this set by $\bar{D}$.

Define

$$
C_{0}=\{\psi \in C: \psi(0) \in \bar{D}\} .
$$

R e mark 2.1. Since $C_{0}$ is a closed subset of the Banach space $C$, it is a complete metric space equipped with the metric $\varrho_{C_{0}}$ given by the formula

$$
\varrho_{C_{0}}\left(\psi_{1}, \psi_{2}\right)=\left\|\psi_{1}-\psi_{2}\right\|_{C}, \quad \psi_{1}, \psi_{2} \in C_{0} .
$$

Let $f:[0, T] \times C \rightarrow X$. We will also need the following assumption: 
Assumption $\left(\mathrm{A}_{2}\right)$. There exists a constant $L>0$ such that

$$
\left\|f\left(s, \psi_{1}\right)-f\left(s, \psi_{2}\right)\right\| \leq L\left\|\psi_{1}-\psi_{2}\right\|_{C} \quad \text { for } s \in[0, T], \psi_{1}, \psi_{2} \in C,
$$

and there exist a continuous nondecreasing function $\omega:[0, \infty) \rightarrow[0, \infty)$ and a continuous function $k:[0, T] \rightarrow X$ such that

$\left\|f\left(s_{1}, \psi\right)-f\left(s_{2}, \psi\right)\right\| \leq \omega\left(\|\psi\|_{C}\right)\left\|k\left(s_{1}\right)-k\left(s_{2}\right)\right\| \quad$ for $s_{1}, s_{2} \in[0, T], \psi \in C$.

3. Auxiliary theorems. Now, we formulate two definitions of weak solutions. The first was given by Evans [6], and the second by Kartsatos and Parrott [8]. Some properties of weak solutions were discussed by Kartsatos in [7].

For a given function $\tilde{f}:[0, T] \rightarrow X$ and $x \in X$, a continuous function $u:[0, T] \rightarrow X$ is said to be a weak solution of the problem

$$
w^{\prime}(t)+A(t) w(t)=\widetilde{f}(t), \quad t \in[0, T], \quad w(0)=x,
$$

if for every $\widetilde{T} \in(0, T]$ there exist a sequence $P^{n}=\left\{0=t_{n 0}<t_{n 1}<\right.$ $\left.\ldots<t_{n N(n)}=T(n)\right\}(n \in \mathbb{N})$ of partitions and sequences $\left\{u_{n j}\right\}_{j=0,1, \ldots, N(n)}$, $\left\{\widetilde{f}_{n j}\right\}_{j=1, \ldots, N(n)}(n \in \mathbb{N})$ of elements in $X$ such that

(i) $\widetilde{T} \leq T(n) \leq T(n \in \mathbb{N})$ and

$$
\lim _{n \rightarrow \infty} \max _{j \in\{1, \ldots, N(n)\}}\left(t_{n j}-t_{n, j-1}\right)=0,
$$

(ii) $u_{n 0}:=x(n \in \mathbb{N})$ and

$$
\frac{u_{n j}-u_{n, j-1}}{t_{n j}-t_{n, j-1}}+A\left(t_{n j}\right) u_{n j}=\widetilde{f}_{n j} \quad(j=1, \ldots, N(n) ; n \in \mathbb{N}),
$$

(iii) $\widetilde{f}_{n}$ is convergent to $\widetilde{f}$ in $L^{1}(0, T ; X)$, where $\widetilde{f}_{n}(t):=\widetilde{f}_{n j}$ for $t \in$ $\left(t_{n, j-1}, t_{n j}\right](j=1, \ldots, N(n) ; n \in \mathbb{N})$, and $u_{n}$ converges uniformly to $u$ on $[0, T]$, where $u_{n}(t):=u_{n j}$ for $t \in\left(t_{n, j-1}, t_{n j}\right](j=1, \ldots, N(n) ; n \in \mathbb{N})$.

For given functions $f:[0, T] \times C \rightarrow X$ and $\phi \in C_{0}$, a continuous function $u:[-r, T] \rightarrow X$ is said to be a weak solution of the problem

$$
w^{\prime}(t)+A(t) w(t)=f\left(t, w_{t}\right), \quad t \in[0, T], \quad w_{0}=\phi,
$$

if $u(t)=\phi(t)$ for $t \in[-r, 0]$ and $u$ is a weak solution of the problem

$$
w^{\prime}(t)+A(t) w(t)=f\left(t, u_{t}\right), \quad t \in[0, T], \quad w(0)=\phi(0) .
$$

Now, we formulate two theorems which are consequences of the results obtained by Kartsatos and Parrott [8].

TheOREM 3.1. Suppose that the operators $A(t), t \in[0, T]$, and the function $f$ satisfy Assumptions $\left(\mathrm{A}_{1}\right)$ and $\left(\mathrm{A}_{2}\right)$. Then for each $\phi \in C_{0}$ there exists 
exactly one weak solution of problem (3.1). Moreover, if $\alpha>L$ is such that, for each $t \in[0, T], A(t)-\alpha I$ is accretive then

$$
\left\|u_{1}(t)-u_{2}(t)\right\| \leq e^{-(\alpha-L) t}\left\|\phi_{1}-\phi_{2}\right\|_{C}, \quad t \in[0, T],
$$

where $u_{i}(i=1,2)$ is the (unique) weak solution of the problem

$$
\begin{gathered}
w^{\prime}(t)+A(t) w(t)=f\left(t, w_{t}\right), \quad t \in[0, T], \\
w_{0}=\phi_{i} \in C_{0} \quad(i=1,2) .
\end{gathered}
$$

Theorem 3.2. Suppose that the operators $A(t), t \in[0, \infty)$, and the function $f:[0, \infty) \times C \rightarrow X$ satisfy Assumptions $\left(\mathrm{A}_{1}\right)$ and $\left(\mathrm{A}_{2}\right)$ on the interval $[0, \infty)$ in place of $[0, T]$. Then for each $\phi \in C_{0}$ there exists exactly one weak solution $u_{\phi}$ of the problem

$$
w^{\prime}(t)+A(t) w(t)=f\left(t, w_{t}\right), \quad t \in[0, \infty), \quad w_{0}=\phi .
$$

Moreover, if $\alpha>L$ is such that, for each $t \in[0, \infty), A(t)-\alpha I$ is accretive then

$$
\left\|u_{1}(t)-u_{2}(t)\right\| \leq e^{-(\alpha-L) t}\left\|\phi_{1}-\phi_{2}\right\|_{C}, \quad t \in[0, \infty),
$$

where $u_{i}(i=1,2)$ is the (unique) weak solution of the problem

$$
\begin{gathered}
w^{\prime}(t)+A(t) w(t)=f\left(t, w_{t}\right), \quad t \in[0, \infty), \\
w_{0}=\phi_{i} \in C_{0} \quad(i=1,2) .
\end{gathered}
$$

Consequently, $u_{\phi}$ is asymptotically stable.

4. Result. Let $g: C \rightarrow C_{0}$. We will need the following assumption:

Assumption $\left(\mathrm{A}_{3}\right)$. There exist constants $M>0$ and $\beta \in \mathbb{R}$ such that

$$
\left\|g\left(w_{\hat{T}}\right)-g\left(\widetilde{w}_{\hat{T}}\right)\right\|_{C} \leq M e^{\beta t_{0}}\|w-\widetilde{w}\|_{C\left(\left[t_{0}, \hat{T}\right], X\right)}
$$

for all $w, \widetilde{w} \in C([-r, T], X)$ and $\widehat{T} \in\left[t_{0}+r, T\right]$.

Now, we present two theorems on weak solutions of nonlocal problems.

Theorem 4.1. Suppose that the operators $A(t), t \in[0, T]$, and the functions $f:[0, T] \times C \rightarrow X$ and $g: C \rightarrow C_{0}$ satisfy Assumptions $\left(\mathrm{A}_{1}\right)$ $\left(\mathrm{A}_{3}\right)$. Moreover, suppose that there is $\alpha>L$ such that, for each $t \in[0, T]$, the operator $A(t)-\alpha I$ is accretive. Then for each $T^{*} \in\left[t_{0}+r, T\right]$, where $t_{0} \in(\max \{0, \ln (M)\} /(\alpha-L-\beta), T-r), \ln (M)<(\alpha-L-\beta)(T-r)$ and $\beta<\alpha-L$, there is a unique $\phi_{*} \in C_{0}$ and exactly one weak solution $u_{*}:[-r, T] \rightarrow X$ of the problem

$$
w^{\prime}(t)+A(t) w(t)=f\left(t, w_{t}\right), \quad t \in[0, T], \quad w_{0}=\phi_{*},
$$

satisfying the condition

$$
\left(u_{*}\right)_{0}=g\left(\left(u_{*}\right)_{T^{*}}\right)=\phi_{*} .
$$


Moreover, for the (unique) weak solution $u_{\phi}$ of the problem

$$
w^{\prime}(t)+A(t) w(t)=f\left(t, w_{t}\right), \quad t \in[0, T], \quad w_{0}=\phi,
$$

where $\phi$ is an arbitrary function belonging to $C_{0}$, the following inequality holds:

$$
\left\|u_{\phi}(t)-u_{*}(t)\right\| \leq e^{-(\alpha-L) t}\left\|\phi-g\left(\left(u_{*}\right)_{T^{*}}\right)\right\|_{C}, \quad t \in[0, T] .
$$

Proof. By Theorem 3.1, there is exactly one weak solution $u_{\phi}:[-r, T]$ $\rightarrow X$ of problem (4.3), where $\phi$ is an arbitrary function belonging to $C_{0}$. Moreover, by Theorem 3.1, for any two functions $\phi_{i} \in C_{0}(i=1,2)$ the (unique) weak solutions $u_{\phi_{i}}(i=1,2)$ of the problems

$$
\begin{gathered}
w^{\prime}(t)+A(t) w(t)=f\left(t, w_{t}\right), \quad t \in[0, T], \\
w_{0}=\phi_{i} \quad(i=1,2),
\end{gathered}
$$

respectively, satisfy the inequality

$$
\left\|u_{\phi_{1}}(t)-u_{\phi_{2}}(t)\right\| \leq e^{-(\alpha-L) t}\left\|\phi_{1}-\phi_{2}\right\|_{C}, \quad t \in[0, T] .
$$

Let $T^{*}$ be an arbitrary number such that $T^{*} \in\left[t_{0}+r, T\right]$, where $t_{0} \in$ $(\max \{0, \ln (M)\} /(\alpha-L-\beta), T-r, \ln (M)<(\alpha-L-\beta)(T-r)$ and $\beta<\alpha-L$.

Next, define a mapping $F_{T^{*}}: C_{0} \rightarrow C_{0}$ by the formula

$$
F_{T^{*}}(\phi)=g\left(\left(u_{\phi}\right)_{T^{*}}\right), \quad \phi \in C_{0} .
$$

Observe that, from Remark 2.1, from (2.1) and (4.6), from Assumption $\left(\mathrm{A}_{3}\right)$, from (4.5) and from the fact that $T^{*} \in\left[t_{0}+r, T\right]$ and $t_{0}>$ $\max \{0, \ln (M)\} /(\alpha-L-\beta)$,

$$
\begin{aligned}
& \varrho_{C_{0}}\left(F_{T^{*}}\left(\phi_{1}\right), F_{T^{*}}\left(\phi_{2}\right)\right)=\left\|F_{T^{*}}\left(\phi_{1}\right)-F_{T^{*}}\left(\phi_{2}\right)\right\|_{C}=\left\|g\left(\left(u_{\phi_{1}}\right)_{T^{*}}\right)-g\left(\left(u_{\phi_{2}}\right)_{T^{*}}\right)\right\|_{C} \\
& \quad \leq M e^{\beta t_{0}}\left\|u_{\phi_{1}}-u_{\phi_{2}}\right\|_{C\left(\left[t_{0}, T^{*}\right], X\right)}=M e^{\beta t_{0}} \sup _{t \in\left[t_{0}, T^{*}\right]}\left\|u_{\phi_{1}}(t)-u_{\phi_{2}}(t)\right\| \\
& \quad \leq M e^{\beta t_{0}} \sup _{t \in\left[t_{0}, T^{*}\right]} e^{-(\alpha-L) t}\left\|\phi_{1}-\phi_{2}\right\|_{C} \\
& \quad \leq M e^{(-\alpha+\beta+L) t_{0}}\left\|\phi_{1}-\phi_{2}\right\|_{C}<\varrho_{C_{0}}\left(\phi_{1}, \phi_{2}\right) \quad \text { for } \phi_{1}, \phi_{2} \in C_{0} .
\end{aligned}
$$

Hence, by the Banach contraction theorem $F_{T^{*}}$ has a unique fixed point $\phi_{*} \in C_{0}$. Moreover, by Theorem 3.1, there exists exactly one weak solution $u_{*}:[-r, T] \rightarrow X$ of problem (4.1). Obviously, condition (4.2) holds.

Finally, Theorem 3.1 implies that

$$
\left\|u_{\phi}(t)-u_{*}(t)\right\| \leq e^{-(\alpha-L) t}\left\|\phi-\phi_{*}\right\|_{C}, \quad t \in[0, T],
$$

where $u_{\phi}$ is the unique weak solution of problem (4.3).

From the above inequality and from (4.2), we have (4.4).

The proof of Theorem 4.1 is complete.

As a consequence of Theorem 3.2 and of an argument similar to the argument from the proof of Theorem 4.1, we obtain the following theorem: 
Theorem 4.2. Suppose that the operators $A(t), t \in[0, \infty)$, and the functions $f:[0, \infty) \times C \rightarrow X$ and $g: C \rightarrow C_{0}$ satisfy Assumptions $\left(\mathrm{A}_{1}\right)-\left(\mathrm{A}_{3}\right)$ on the interval $[0, \infty)$ in place of $[0, T]$. Moreover, suppose that there is $\alpha>L$ such that, for each $t \in[0, \infty)$, the operator $A(t)-\alpha I$ is accretive. Then for each $T^{*}>t_{0}+r$, where $t_{0}>\max \{0, \ln (M)\} /(\alpha-L-\beta)$ and $\beta<\alpha-L$, there is a unique $\phi_{*} \in C_{0}$ and exactly one weak solution $u_{*}:[-r, \infty) \rightarrow X$ of the problem

$$
w^{\prime}(t)+A(t) w(t)=f\left(t, w_{t}\right), \quad t \in[0, \infty), \quad w_{0}=\phi_{*},
$$

satisfying the condition

$$
\left(u_{*}\right)_{0}=g\left(\left(u_{*}\right)_{T^{*}}\right)=\phi_{*} .
$$

Moreover, for the (unique) weak solution $u_{\phi}$ of the problem

$$
w^{\prime}(t)+A(t) w(t)=f\left(t, w_{t}\right), \quad t \in[0, \infty), \quad w_{0}=\phi,
$$

where $\phi$ is an arbitrary function belonging to $C_{0}$, the following inequality holds:

$$
\left\|u_{\phi}(t)-u_{*}(t)\right\| \leq e^{-(\alpha-L) t}\left\|\phi-g\left(\left(u_{*}\right)_{T^{*}}\right)\right\|_{C}, \quad t \in[0, \infty) .
$$

Consequently, $u_{*}$ is asymptotically stable.

$\mathrm{R}$ e mark 4.1. Let $g$ be a function defined by the formula

$$
g(\psi)=M e^{\beta t_{0}} \psi \quad \text { for } \psi \in C,
$$

where $M>0, \beta<\alpha-L, \alpha>L, \ln (M)<(\alpha-L-\beta)(T-r)$ ( $L$ is the constant from Assumption $\left.\left(\mathrm{A}_{3}\right)\right)$ and $t_{0} \in(\max \{0, \ln (M)\} /(\alpha-L-\beta), T-r)$.

If the following condition holds:

$$
\psi \in C \Rightarrow M e^{\beta t_{0}} \psi(0) \in \bar{D}
$$

then $g: C \rightarrow C_{0}$.

Observe that

$$
\begin{aligned}
\left\|g\left(w_{\hat{T}}\right)-g\left(\widetilde{w}_{\hat{T}}\right)\right\|_{C} & =M e^{\beta t_{0}}\left\|w_{\hat{T}}-\widetilde{w}_{\hat{T}}\right\|_{C}=M e^{\beta t_{0}} \sup _{t \in[-r, 0]}\left\|w_{\hat{T}}(t)-\widetilde{w}_{\hat{T}}(t)\right\| \\
& =M e^{\beta t_{0}} \sup _{t \in[-r, 0]}\|w(t+\widehat{T})-\widetilde{w}(t+\widehat{T})\| \\
& \leq M e^{\beta t_{0}}\|w-\widetilde{w}\|_{C\left(\left[t_{0}, \hat{T}\right], X\right)}
\end{aligned}
$$

for all $w, \widetilde{w} \in C([-r, T], X)$ and $\widehat{T} \in\left[t_{0}+r, T\right]$.

Consequently, $g$ satisfies Assumption $\left(\mathrm{A}_{3}\right)$ and Theorem 4.1 can be applied if the other assumptions are satisfied. In particular, for each $T^{*} \in$ $\left[t_{0}+r, T\right]$ the nonlocal condition (4.2) is of the form

$$
u_{*}(t)=M e^{\beta t_{0}} u_{*}\left(t+T^{*}\right) \quad \text { for } t \in[-r, 0] .
$$

It is easy to see that if the interval $[0, T]$ is replaced by $[0, \infty)$ in $(4.7)$ then $g$ satisfies Assumption $\left(\mathrm{A}_{3}\right)$ on $[0, \infty)$ and Theorem 4.2 can be applied 
if $M, \beta$ and $t_{0}$ satisfy the suitable assumptions of Theorem 4.2. Moreover, the nonlocal condition (4.2) is of the form (4.8).

Re mark 4.2. Let $g$ be a function defined by the formula

$$
(g(\psi))(t)=\frac{M e^{\beta t_{0}}}{r} \int_{-r}^{t} \psi(\tau) d \tau \quad \text { for } \psi \in C, t \in[-r, 0],
$$

where $M>0, \beta<\alpha-L, \alpha>L, \ln (M)<(\alpha-L-\beta)(T-r)(L$ is the constant from Assumption $\left.\left(\mathrm{A}_{3}\right)\right)$ and $t_{0} \in(\max \{0, \ln (M)\} /(\alpha-L-\beta), T-r)$.

If the following condition holds:

$$
\psi \in C \Rightarrow \frac{M e^{\beta t_{0}}}{r} \int_{-r}^{0} \psi(\tau) d \tau \in \bar{D}
$$

then $g: C \rightarrow C_{0}$.

Observe that

$$
\begin{aligned}
\left\|g\left(w_{\hat{T}}\right)-g\left(\widetilde{w}_{\hat{T}}\right)\right\|_{C} & =\sup _{t \in[-r, 0]}\left\|\left(g\left(w_{\hat{T}}\right)\right)(t)-\left(g\left(\widetilde{w}_{\hat{T}}\right)\right)(t)\right\| \\
& =\frac{M e^{\beta t_{0}}}{r} \sup _{t \in[-r, 0]}\left\|\int_{-r}^{t}\left[w_{\hat{T}}(\tau)-\widetilde{w}_{\hat{T}}(\tau)\right] d \tau\right\| \\
& =\frac{M e^{\beta t_{0}}}{r} \sup _{t \in[-r, 0]}\left\|\int_{-r}^{t}[w(\tau+\hat{T})-\widetilde{w}(\tau+\hat{T})] d \tau\right\| \\
& \leq M e^{\beta t_{0}} \sup _{t \in[-r, 0]} \sup _{\tau \in[-r, t]}\|w(\tau+\widehat{T})-\widetilde{w}(\tau+\widehat{T})\| \\
& \leq M e^{\beta t_{0}} \sup _{\tau \in[-r, 0]}\|w(\tau+\widehat{T})-\widetilde{w}(\tau+\widehat{T})\| \\
& \leq M e^{\beta t_{0}}\|w-\widetilde{w}\|_{C\left(\left[t_{0}, \hat{T}\right], X\right)}
\end{aligned}
$$

for all $w, \widetilde{w} \in C([-r, T], X)$ and $\widehat{T} \in\left[t_{0}+r, T\right]$.

Consequently, $g$ satisfies Assumption $\left(\mathrm{A}_{3}\right)$ and Theorem 4.1 can be applied if the other assumptions are satisfied. In particular, for each $T^{*} \in$ $\left[t_{0}+r, T\right]$ the nonlocal condition (4.2) is of the form

$$
u_{*}(t)=\frac{M e^{\beta t_{0}}}{r} \int_{-r}^{t} u_{*}\left(\tau+T^{*}\right) d \tau \quad \text { for } t \in[-r, 0] .
$$

It is easy to see that if the interval $[0, T]$ is replaced by $[0, \infty)$ in $(4.9)$ then $g$ satisfies Assumption $\left(\mathrm{A}_{3}\right)$ on $[0, \infty)$ and Theorem 4.2 can be applied if $M, \beta$ and $t_{0}$ satisfy the suitable assumptions of Theorem 4.2. Moreover, the nonlocal condition (4.2) is of the form (4.10). 


\section{References}

[1] J. Bochenek, An abstract semilinear first order differential equation in the hyperbolic case, Ann. Polon. Math. 61 (1995), 13-23.

[2] L. Byszewski, Theorems about the existence and uniqueness of solutions of a semilinear evolution nonlocal Cauchy problem, J. Math. Anal. Appl. 162 (1991), 494-505.

[3] - Uniqueness criterion for solution of abstract nonlocal Cauchy problem, J. Appl. Math. Stochastic Anal. 6 (1993), 49-54.

[4] - Existence and uniqueness of mild and classical solutions of semilinear functionaldifferential evolution nonlocal Cauchy problem, in: Selected Problems of Mathematics, Cracow University of Technology, Anniversary Issue 6 (1995), 25-33.

[5] M. Crandall and A. Pazy, Nonlinear evolution equations in Banach spaces, Israel J. Math. 11 (1972), 57-94.

[6] L. Evans, Nonlinear evolution equations in an arbitrary Banach space, ibid. 26 (1977), 1-42.

[7] A. Kartsatos, A direct method for the existence of evolution operators associated with functional evolutions in general Banach spaces, Funkcial. Ekvac. 31 (1988), 89-102.

[8] A. Kartsatos and M. Parrott, A simplified approach to the existence and stability problem of a functional evolution equation in a general Banach space, in: Infinite Dimensional Systems, (F. Kappel and W. Schappacher (eds.), Lecture Notes in Math. 1076, Springer, Berlin, 1984, 115-122.

[9] T. Winiarska, Parabolic equations with coefficients depending on t and parameters, Ann. Polon. Math. 51 (1990), 325-339.

[10] - Regularity of solutions of parabolic equations with coefficients depending on $t$ and parameters, ibid. 56 (1992), 311-317.

Institute of Mathematics

Cracow University of Technology

Warszawska 24

31-155 Kraków, Poland

E-mail: lbyszews@usk.pk.edu.pl

Reçu par la Rédaction le 8.11.1995

Révisé le 15.6.1996 